PRÉSIDENT DE SÉANCE : A. G. WILSON.

\title{
PR0BLÈMES DE NORMALISATION
}

WiLson. - In the report of the working-group of Sub-Commission $9 b$, several recommendations are made. On page 23 of their report the working group recommends that :

1. scales be specified for the various quantities to be measured;

2. equipment suitable for field work be developed and construction standards for this equipment be specified;

3. scales be correlated whenever one and the same quantity may be measured by different methods,

and finally, the recommendation was made that a Symposium be held, in order to discuss and act on these recommendations.

This is that symposium and this afternoon has been set aside for these discussions. Initially, Prof. Coutrez was to present the problems of standardization, but unfortunately he was unable to be here. However, in order that the Symposium be of aid to the working group, this afternoon we should give our ideas and advice with regard to the matters covered by their recommendations. I use the word advice here, since our discussions this afternoon will be only informal and will in no sense constitute formal resolutions. Indeed, I shall not be surprised if some of our advice is conflicting. The discussions of the past week have pointed out to all of us that in some areas we are not ready to go on record in final form. There is still much work to be done. In order to profit by everyone's experience and ideas, we should keep this an open discussion, but an open discussion must have some sort of structure. In order to meet this problem in the absence of Dr. Coutrez, Dr. Rösch appointed a committee to make some suggestions regarding the problems of standardization. There is an old American saying that a committee consists of a group of men who individually can do nothing but who can join together and collectively make a decision that nothing can be done. And this is why you have before you a set of questions instead of a set of suggestions. These questions parallel the recommendations of Sub-Commission $9 \mathrm{~b}$, so this afternoon we should perhaps focus 
primarily of the questions on the list which has been submitted by the committee :

I. Definitions and normalization problems of image quality. Parameters of image structure. Integrated quality of images. Time resolution of image quality. Normalization of solar seeing.

II. Standardization of instruments for measuring image quality. Types of instruments.

Problems of intercomparison.

Accuracy requirements in seeing measurements.

Techniques for predicting large telescope seeing from small instrument observations.

III. Normalization of sky brightness and transparency observations.

IV. Frequency and duration of site testing observations.

Sampling problems.

V. Problems of data reduction and comparison.

Desiderata in data presentation and comparison.

Treatment of existing data.

However, there are certainly a great many other questions we should discuss, perhaps as important as those on the list. If there are other questions which anyone feels should be added, I would invite you to bring them to my attention during the coffee break.

It is most important for us to consider the several audiences who will be interested in the report of this Symposium. There are several groups who stand to gain standardization in different ways. First, there are those primarily interested in the physics of seeing. They are well represented here. Then there are those concerned with engineering and design studies. It was pointed out earlier in the Symposium that there are economic problems in balancing the gains in seeing against costs of increasing telescope size. Solution of these problems would be aided greatly by standardized and comparable measures of seeing at various sites. There are also those concerned with selecting the best site for their new telescope. There are many such groups who do not choose to become specialists in the physics of seeing but who would profit by standardization and the advice of this Symposium. These groups are an important part of the audience of our report.

Regardless of the desirability of standardization from the point of view of the needs of several groups, it is not clear to the committee, and I am sure all of us have doubts, whether standardization at this time is possible. We shall perhaps find several areas in which we are 
not going to reach agreement, and even where finally there is agreement, it does not necessarily mean that we have the final answer. It is therefore important to bear in mind that what we come up with here should not necessarily be crystallised at the present time. We must be free to make changes as experience accumulates. However, the danger is that once something has appeared in a publication, there is a sort of " aura of print" which dignifies it with a credibility which it may not deserve. So, especially in print, it is important that we stress the tentative nature of some of our advice.

What then may we seek as the specific products of our discussion this afternoon? We shall perhaps not have, as specific products, a set of hard and fast recommendations, but I think we shall have made a great deal of progress if we come up with more precise formulations of our problems and a disclosure of questions needing additional research.

Let us turn to the first section of the question list : Definitions of Normalization Problems having to do with image quality, or seeing. I would like to call first on Dr. Meinel.

Meinel. - In speaking about a subject as broad as standards and normalization, it is a bit hazardous to be the first to speak; nevertheless I will attempt this task and then retire to my chair. First, I would like to make it clear that I will deal with the problem of site testing as contrasted to the study of seeing at an existing observatory. The two are quite different problems.

To start, I would first like to divide site investigations into three general classes, beginning with the simplest type of investigation and ending with the most complex deemed feasible at this time. The classes are as follows :

Class III. - A class III site survey would be one done with small visual telescopes of single aperture less than i2-inches. The phase of the Palomar survey in which 4-inch refractors were used for ring and motion tests, would be in this class.

Class II. - A class II site survey would be one done with visual multiple-beam instruments wherein the relative motion of the images through different air paths is observed. The degree of confidence would be such that a survey with this class of instrument would be considered indicative of the seeing with a large telescope.

Class I. - A class I site survey would be one done with an impersonal instrument which integrates image motion either with a single beam or multiple beam. Such an instrument should give reliable indication of the performance of a large telescope at the site, subject to the limitation that the two be at the same absolute elevation above the local terrain. 
The preceeding three classes apply to instruments in the strict sense rather than to site surveys, since most site surveys will in actuality proceed from class III to I during the course of the programme unless the programme is terminated by other conditions. This fact arises from the usual procedure of starting a site testing programme as soon as it appears likely that a new observatory is to be established. To date, the only instruments readily at hand are small portable instruments. On the few site testing programme that have attained a class II or I rating, the instruments have been developed specifically for the job. Perhaps this situation will change as more class II and I instruments come into existence.

The degree of confidence in the results of a site survey depends not only on the instrument class, but also upon the programme of observation. Seeing is a phenomenon that varies over a wide range at any one site. Since the differences in seeing at reasonable astronomical locations are hoped to be small, one must continue observations for rather a long time in order for the difference between the sites to be statistically significant. I would therefore suggest that the class of a site survey be associated in some manner with the frequency of testing at a site. In other words, a transient or " spot-check" type of site testing should rank one class lower than would a semi-permanent type of testing. A class I instrument used for spot-checking of various sites would therefore rank as a class II survey unless the accumulated number of nights at each site exceeds a certain number, say roo nights.

Returning to the general question of the classes, class III instruments, while widely used to date, do not appear to be reliable in the hands of the personnel usually available for these programmes. To be specific, the Danjon test has been shown to be usable in the hands of a skilled astronomer, but in the hands of test personnel it generally indicates better seeing than one finds when a telescope is later built. The explanation may be that the observer is unable to isolate superimposed effects. Diffraction ring visibility is hard to use, since the seeing almost always is such that the diffraction rings from a 6-inch telescope are visible. Image motion is also hard to assess with a single aperture telescope since the observer may tend to ascribe the motion he sees to wind shake of the instrument rather than to the seeing. Double-beam systems, such as that described by Stock, tend to eliminate this effect and therefore enhance the degree of confidence of the results.

When one uses a class I instrument for the measurement of seeing, it is necessary to define what we call the " image diameter ". For the sake of argument, let us assume that in the seeing disc, the distribution of intensity with radius from the centre of the image is of the generalized Voigt type for a large telescope, or with time integration with a smaller telescope. I would suggest that the image diameter is that 
diameter $D_{s u}$ which contains $80 \%$ of the total light energy in the vicinity of the focal point of the instrument. Dr. Hoag reports that his simple knife-edge method actually defines nearer to $90 \%$, and I would not be averse to adopting this value in general for the more sophisticated impersonal methods, such as have been described by Dr. Hogg.

We must further specify the integration time when using an impersonal instrument in the determination of the image diameter. Again for the sake of a number, let us specify that the seeing disc image diameter be determined with a minimum of $\mathrm{I} s$ integration time. This means that if the seeing disturbance has a drift velocity of $3 \mathrm{~m} / \mathrm{s}$, a $15-\mathrm{cm}$ aperture telescope will effectively integrate the seeing over an area $1 \check{\partial} \times 3$ oo $\mathrm{cm}$.

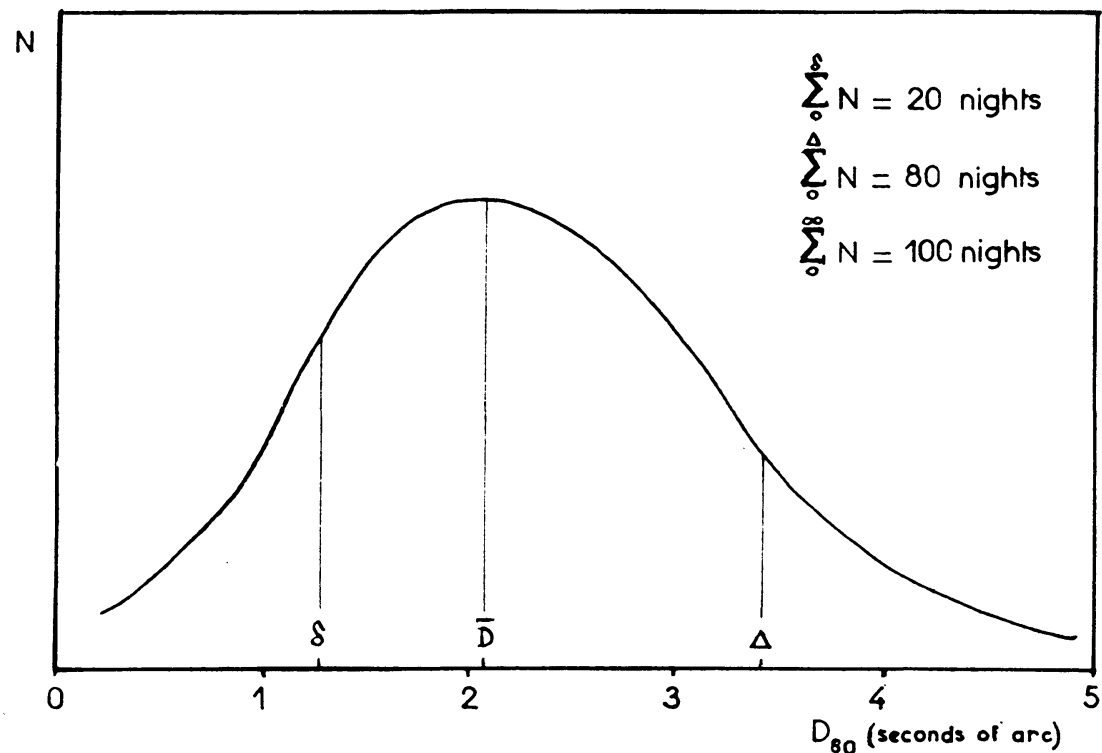

Fig. 74. - Illustration of a typical frequency function $\mathrm{N}$ for the image diameter $\mathbf{D}_{\mathbf{8 0}}$.

In summarizing the performance of a site one must specify the frequency of occurrence of seeing of various image sizes ; standards must be defined if we are to be able to compare the relative performance of several sites. Let us assume that the distribution of seeing is as shown in figure 74. The first parameter to specify the curve would be the seeing disc size at the maximum of the frequency function, $\overline{\mathrm{D}}$. The other parameters are the values $\delta$ and $\Delta$ of the image size for the $20 \%$ fraction of the best seeing and for the $20 \%$ fraction of the poorest seeing. These three values are of practical importance in the assessment of a site for various types of research programme. While it is true that 
important advances can often be made only with the very best seeing, practical programmes require a site with reasonably good seeing most of the time. For example, spectroscopic programmes function equally well with any seeing better than about 1.5 seconds of arc. Photographic programmes, on the other hand, especially when field correctors are used, operate at about equal efficiency with any seeing better than about 0.5 seconds of arc.

The final criterion that is important in specifying the performance of a site is the number of nights used in determining the frequency function of the image sizes. In general this number is less than the total number of useable nights during the period of observation, due to practical problems encountered on: any site survey. The total number of useable nights at a site is generally known from meteorological data and could be used for the determination of the absolute frequency function of image diameters. The final decision between a site with relatively few useable nights but of good seeing, and one with many nights of poorer seeing, is one that will continue to tax the wisdom of the persons who in the final analysis must select the site for an observatory.

Siedentopf. - I would propose to add a class $O$ that includes the paramount observations with great instruments, say with mirrors with $2 \mathrm{~m}$ diameter upward.

Scorer. - I think it is a very good idea. The cause of the trouble is in the atmosphere, and you want to know something more about what is going on there. Information collected with the best possible instruments will form a much better basis for a proper understanding. At this point I would like to ask at what class is it possible to measure the height at which the disturbance exists.

Protheroe. - This sort of thing can be done as a routine with class II.

Scorer. - This is the kind of statistic that would be extremely useful. It is not enough for a full understanding simply to say we have got a certain kind of seeing; if you can differentiate between the effects from different levels of the atmosphere this will be extremely helpful in the long run.

WiLson. - It is well to emphasize that even though class II is primarily for site surveys, it could also give very useful information on the physics of seeing.

Meinel. - I should reiterate that my discussion of standards was with regard to the problem of site testing rather than to the important question of the physics of seeing. Dr. Siedentopf's remarks concerning the large existing telescopes is correct, but one is indeed fortunate if one can explore a new site with a telescope larger than 16 to 24 inches aperture, since the costs become rapidly prohibitive. 
VAN IsAcker. - To understand the physics of seeing, a large instrument would not be suitable because of space integration. A better one will be a double beam telescope with small aperture, the best being an interferometer.

Rösch. - I would like to mention at this point that the working group on site testing has already recommended that experiments should be made at the existing observatories with large instruments and with site testing equipment simultaneously in order to make comparisons between observatory and field instruments; we are also confronted with some normalisation problems with the large instruments. Another point which I wanted to mention was that, as Prof. Scorer said, it would be extremely interesting to know the elevation of the disturbing layers, but we must be extremely careful when we try to deduce the height of these layers by optical observations. Some of the observations proposed, for instance focusing on the limb of the Moon, appear to give completely wrong results.

VAN ISACKER. - I do not agree that a large instrument will give more information about the physics of the problem, because it gives in fact an integration over the whole aperture; it cannot give much information on small-scale phenomena. I suggest that the best instrument will be a double beam instrument with two small apertures. The best thing to measure, I suppose, is the difference in phase from the incoming beam.

Scorer. - I was basing my remarks on the fact that Dr. Protheroe convinced me yesterday, that it is quite feasible to measure the height with a double beam instrument. Is not this so ? It seems to me that it might be feasible by observing single and double stars.

Wilson. - Any more comments on the class I analysis? Then class II or III.

Courtès. - About the question of human organisation I think it is more important to have impersonal instruments for class III or II than for class I; class I is needed only for a few sites, to which a skilled astronomer-optician can come often to see the real quality of the images. We can use a 2o-inch telescope; that is quite enough to judge quality, especially if it is completed by some double-beam method.

Bowen. - It seems to be very desirable in class I also to specify not $3 \mathrm{~m}$, but an elevation approximately equal to the height of the instruments that you are seeking the site for; in view of the Lynds results it seems to me that $3 \mathrm{~m}$ is altogether too low.

Miss Underhill. - I just wish to enquire whether with class I type of instruments you envisage developping an impersonal analysis system 
to deal with the vast body of data obtained? The difference between this type of instrument and a visually used instrument is that a visually used instrument comes equipped with its own computer, that is to say a human being who does complicated, weighted integrations of the data. It is not clear whether the term " class I " includes the reduction process.

Scorer. - With regard to this $3 \mathrm{~m}$ height, I think we might learn something here from the standards laid down for meteorological instruments. They specify not merely height above the ground but also the nature of the terrain in the immediate vicinity. Now, in this case the relevant height above the ground may be different for different sites and so some sort of judgement must be used.

With regard to the $\mathrm{I}$-second time interval : this is a time which has been arbitrarily specified in an innocent sort of way. The time which should be specified would seem to be something like the diameter of the telescope divided by the velocity with which the disturbance crosses the telescope. Is one second on arbitrary time, or what is it based on ?

Wilson. - One suggestion was that the characteristic time was the time needed to reach the asymptotic value of image size, but there may be some other characteristic time which would be more useful for the physics of seeing. Has anyone any comments to make on this point?

Siedentopf. - The spectrum of the seeing effect is quite well known from American observations and from our own. It contains frequencies up to about 20 or $30 \mathrm{c} / \mathrm{s}$, with a maximum near $\mathrm{r} / \mathrm{c} / \mathrm{s}$. This is quite distinct from the spectrum of the intensity fluctuations, of the scintillation, on where much higher frequencies occur. If we have to think of these low frequencies, it seems that a longer integration time is necessary, since with i $s$ we integrate only over, say, ro c/s on the average.

Rösch. - May I ask Prof. Siedentopf what he means by the spectrum of seeing which is different from the spectrum of scintillation ? I understand quite well. what is the spectrum of scintillation, because scintillation is the fluctuation of the total intensity and I understand what you mean by the spectrum of the frequencies of a certain quantity. But what is the quantity you call the seeing?

Siedentopf. - The image motion.

Rösch. - It would be better to call it image motion, and then everybody would understand what it is. Now I agree. Thank you.

WiLson. - I would like to pass on now to the other parameters of image quality. 
Courtès. - I think we have not to forget that if in future years we make more and more use of the image converter or electronic camera, exceptional seeing even during short exposures will be very useful with a large instrument. The visual estimation of the resolving power in a very large instrument is according to our general experience about 0.3 seconds of arc and this determines our first need to find a sitetesting method able to detect small deviations of this order of magnitude. In fact, if you consider the general surface of your telescope when you take an instantaneous picture for a few milliseconds during the knife-edge test, the surface that I called $\Gamma$ has to be absolutely flat for a diameter of at least $70 \mathrm{~cm}$ if you want to reach 0.2 seconds of arc. The second need is to know also if the small fluctuations of the wave surface have not some very high frequency structure, because, in that case, it will give a series of small spots in the plane of the diaphragm. In the focal plane something like a general scattering of the light occurs and this gives very bad image structure. Then the third need of a survey is to be capable to see if there are different tilts of the large $\Gamma$ elements, and that can be given, for instance, by a method using two beams.

About the question of the flatness of each of these flat surfaces, I think everybody agrees that the diffraction ring method with a ro or 15 -inch instrument is quite good and a very easy to use. For the size of the $\Gamma$ surfaces I think it would not be difficult to imagine a simplified Hartmann system; it could have two beams only, but more than two would be better, and that is not very difficult. After that, the general information about the different parameters will be obtained with class I instruments, a sufficiently large telescope (20-inch) with good guiding. If we do not forget these three points, we can imagine very simple instruments.

HoAG. - I would like to endorse the remarks Dr. Courtès has made concerning the relative degree of coherence in the wave front at the objective. We have, for the most part, been discussing integrated image quality but we are here concerned with the time resolution of image quality. Dr. Rösch's photograph of solar granulation, which demonstrated that the full resolving power of a $38 \mathrm{~cm}$ aperture was attained for one exposure, illustrated the importance of the time resolution of image quality. It is important that class $I$ instruments be designed to measure the frequency of occurrence of relatively large areas of coherence in the wave front.

Rösch. - May I try to clarify the considerations about the wave front, the diffraction ring method and the Hartmann method, in the following way. I think we all agree that the concept of the wave front arriving at the objective is a useful one. But we cannot set up a 3-metre 
telescope anywhere we like, nor can we move it around easily. At only a few places in the world can we hope to obtain all the information which is collected over such a surface. However, what we can do is to use a sampling method as considered in the theory of information. Suppose we use a $25-\mathrm{cm}$ portable telescope; it will give us all the information over its surface. What about larger elements ? We cannot have a large instrument anywhere we like but we can have a system of small mirrors distributed over a large area, in place of the holes used in Hartmann's test. From my experience, this is not easy to adjust, but it is quite feasible. If you can measure the tilt of the wave front on each of these mirrors, you have in fact sampling points all over the wave front. Then the theorem of Shannon tells us that we can estimate the spatial frequencies on the surface up to one-half that corresponding to the interval between the mirrors. Let us draw at least a one-dimensional cross-section of the wave front, and consider its spatial spectrum, i. e. the amplitude of the path difference as a function of the frequency. About the high frequencies, we can obtain full information by using a full mirror of moderate size. From a system of small mirrors, we may obtain information about low frequencies (i. e. long spatial wave-lengths); so we have two parts of the spectrum with a gap in between. If we choose the spacing of the mirrors such that this gap is not too wide, by combining a telescope of moderate size using Danjon's diffraction pattern method and a set of mirrors working as a Hartmann's test, we can get almost full information over a large area of the wave front $(f i g .75)$.

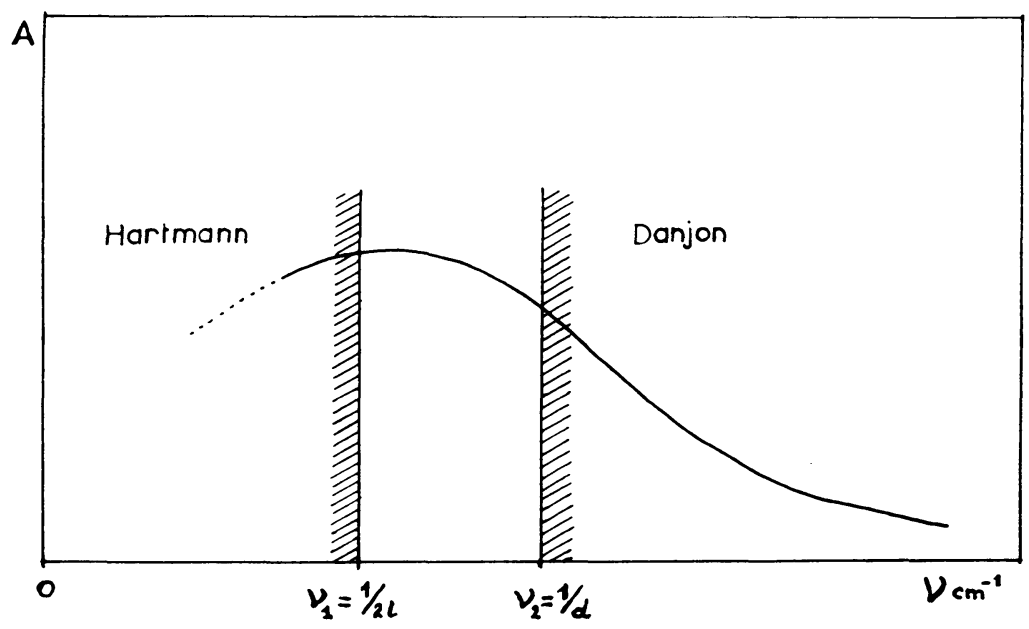

Fig. 75. - Spatial frequency coverage by Hartmann-like method (mirror spacing $l$ ) and Danjon's method (telescope diameter $d$ ); $\nu$, spatial frequency;

$A$, amplitude of the path difference. 
Meinel. - Is it not necessary to construct your network of mirrors to the order of a fraction of a wave-length ? Otherwise, they could change during your observation time and introduce an unknown phase shift from point to point on your diagram.

Rösch. - No, you do not need a phase shift adjustment of the order of wave-lengths, because you are dealing with Hartmann's images which are independent of one another and you do not have to consider the coherence between the different small mirrors; you do not even need to have a regular pattern of images. Provided the mirrors are not moving with respect to one another, you can take a number of pictures with a long exposure and low sensitivity, and that gives you an average; then you just measure the deviations from this average. There is no interference between the various beams because they are not coherent, and this makes the experiment much easier.

Siedentopf. - Perhaps one more remark to clarify the situation. We can compute an image diameter through the mean square fluctuation of the deviation of the rays, either at a given moment over all the elements of a large collecting surface, or over an interval of time on a small fixed collecting surface. Our essential supposition in using small instruments and taking means over the time for evaluating what happens with large instruments, where we take the mean over the surface, is that these two values for the image diameter are essentially the same. This assumption cannot be entirely right since there is a correlation of the deviations over the surface and since the eddies that move over the surface and cause these deviations have only a limited life, so we have movement of a pattern which changes in time. For that reason, it is not quite correct to put these two means equal, but it seems to be nearly correct.

STock. - If we imagine a system of mirrors in which the light reflected from each mirror is collected at one point, or very nearly at one point, then we get a device that looks very much like a telescope, with a long tube, except that in place of one large mirror, there is a system of small ones. If you make it just a one-dimensional instrument of course it is a much simpler piece of equipment.

Rösch. - I would like to reply to Dr. Stock that I have already sketched such a type of instrument with many small mirrors; I shall not enter into the details but just mention that it could have a tube no more than $60 \mathrm{~cm}$ long, the diameter being larger than that. Nothing like a telescope tube.

Wilson. - We should pass now to the last part of the first section on normalisation problems with regard to solar seeing. 
Kiepenheuer. - Because of the existence of strong ground convection in day time, all solar seeing tests should be made at least 15 to $20 \mathrm{~m}$ above ground. Unshielded steel towers (with little surface for the wind) are to be recommended for this purpose. Domes, huts with open roofs, etc. should be avoided.

In order to arrive at a correct diagnosis of the various atmospheric effects, a telescopic aperture of only io to $15 \mathrm{~cm}$ should be used. Blurring, motion and distorsion of the image should be estimated visually according to the scale given in my paper on Solar Site testing in this Symposium. It is advisable however to compare these personal estimates from time to time with impersonal measurements. A description of suitable photoelectric equipment is given in the above paper.

Courtès. - Excuse me asking you a question about solar seeing, but I have noticed, on very short exposures of the Sun, something like a large-scale network, distinct from the Sun's granulation. I think this is certainly caused by the classical distortion of the seeing, due to the rays not coming through the same part of the disturbing layers.

The cells of this network are sometimes quite conspicuous, because the regular granulation of the Sun itself plays the role of a periodic object with a higher spatial frequency than that of the perturbing layer. May be it offers another way of measuring the dimensions of the turbulent cells, during day time, of course!

Rösch. - This is quite true. This phenomenon is the so-called réseau photosphérique discovered by Janssen in the last century on his magnificent granulation photographs. All over the Sun there are regions, which may cover a number of minutes of arc, where the granules appear extremely distinct, whereas they do not appear so in between these regions. This gives the appearance of a sort of network of fuzzy regions (plate XIII). Janssen believed that to be a solar phenomenon, but when we took series of pictures at 20 frames per second, we found how the pattern changed in one-twentieth of a second. That is proof of its atmospheric, not solar origin. The mesh of the network corresponds to the distance between neighbouring homogeneous air masses.

Pierce. - Prof. Kiepenheuer has given us a very excellent tool, namely a tower which is relatively cheap and which can be easily moved about. I think this is of use not only in solar work but also in stellar observations and I would highly recommend it. Parallelogram action is of course of importance. Now, there has been no discussion on the reduction of measurements to the zenith. In other words, if observations are made close to the horizon, as is often done with the sun in the early morning, do we reduce them all to zenith values or not ? 
Wilson. - Thank you for this additional question which perhaps can be discussed under point 5 , data reduction.

May I ask a question? Is there any way of determining the heights of daytime turbulence layers from observations on the Sun?

Kiepenheuer. - The easiest way seems to be to have several telescopes working at different heights on the same tower. That will give you always the upper limit of this convective layer, close to the ground. But the cheapest method is of course that of Dr. Lynds : putting up thermometers and recording temperatures in daytime. This is more difficult than at night, because the thermometers have to be shielded against radiation, but he told me that such equipment is in preparation.

Wilson. - If there are no other comments or discussion, I would like next to take up discussions on part 2, Standardization of Instruments for measuring Image Quality.

Several intriguing instruments were described the other day especially in Dr. Courtès' paper summarizing this subject, but it is an open question whether are we ready to advise on standardization of instruments. If we do not raise the matter of standardization, we may perhaps talk about some of the desirable features of instruments for measuring image quality.

Dommanget. - I have not very much to say about the standardization of instruments and methods of reduction to be used during a site survey. I just want to mention some conditions that in my opinion they should satisfy, especially in the first stages of such a survey.

First of all, the instrument must be as simple and light as possible so that one may be able to move it easily and quickly, in flat regions as well as in mountains, following the general considerations I gave this morning on survey organization.

Secondly, it must be possible to direct the instrument in all azimuths and elevations, because orographic effects varying with the azimuth are observed at numerous stations. I remember for instance, the effect of the Swartberg Mountain observed at Prince Albert (South Africa).

Thirdly, it is of great importance that the method of observation should afford the possibility of making a complete set of observations in all directions, in a short interval of time, in order to eliminate the effects of changes that may occur in the image quality during the night (alteration, image motion or scintillation). Otherwise, if such effects take place during a set of observations, they may mask for instance important orographic effects.

The fourth point concerns the reduction of the observations. This reduction has to be made quickly on the spot itself so that the results of the observations may be taken into account in planning further 
research in the area. In particular, this point is of great importance when some preliminary tests around the site are needed to define more accurately the orographic effects.

A fifth point concerns the accuracy and scale requirements in " seeing " measurements. Two days ago, Dr. Bowen told us that when the image diameter given by a big telescope (Mount Palomar), is of 5 to 6 seconds of arc, the observing conditions may be considered as very bad. The image radius is then roughly 3 seconds of arc. In my opinion, the scale of observation must be limited at least to these 3 seconds of arc for the maximum deviation of the different parts of the wave surface compared to the mean position of this wave in front of the objective. Thus each method of observation proposed for site survey should be adjusted to a scale whose upper limit is about 3 seconds of arc, or perhaps less. Any other method seems useless to me for site survey.

Now, there is a last point that I want to mention. It concerns the correlation between what we observe later on with a big telescope eventually built on the same spot. There are many different opinions on this subject but we need a decisive answer to this fundamental question. I think that only observations made with instruments of these different types, in existing observatories and under different meteorological conditions, following agreed rules, may lead to some knowledge on this point.

Courtès. - There is something that I have not clearly understood concerning the measurement of image deviation by photoelectric variations through a slit. For a 5-metre focal length, I second of arc corresponds to $25 \mu$, and we need a high accuracy to know if the deviations are about one-fourth of this value, that is to say about $6 \mu$. May I ask the opticians present here, if they believe that is is possible, with a 5-metre focal length to get an accuracy better than $6 \mu$.

Rösch. - The diagrams that you have seen have been obtained with $18 \mathrm{~m}$ focal length, but $\mathrm{I}$ think that $\mathrm{I} 5 \mathrm{~m}$ would be a minimum.

Courtès. - It needs a certain aperture too, because if you have an aperture of about 15 or $20 \mathrm{~cm}$, the diameter of your central spot of the diffraction image is about 2 seconds of arc, and there is also the question : is it possible to detect, with the short time constant of the electronic equipment, deviations of about one eight of this value of $2^{\prime \prime}$ ?

Rösch. - I think it is possible to appreciate a motion of a fraction of a second of arc even if the image diameter is more than $r$ second of arc. The relative change is smaller, but it can be measured.

Courtès. - Yes, of course, but I think it needs at least a diameter of about ro inches, and at least a ro-metre focal length. I am afraid 
that, if one uses a smaller instrument, information will be very difficult to interpret. In particular, how it is possible to separate the effect of scintillation, in the case of a small reflector? It needs two cells in that case.

WiLson. - I would like to return briefly to one of the points made by Dr. Dommanget, the importance of being able to observe effects in azimuth. Does this have any bearing on the class instruments which observe only Polaris? Is this a safe procedure for a class III survey? A great many surveys are, of course, conducted with the Polaris instrument for the convenience of the guiding.

Dommanget. - This may be too risky.

WiLson. - May we next consider the problems of intercomparison ? There are two parts to this question : $1^{0}$ Intercomparison of different class III instruments, as for example one site survey with another, and $2^{\circ}$ the problem of predicting the image quality in large telescopes from intercomparisons between classes of instruments. Is it important to standardize class III instruments ?

STock. - The importance of type III instruments will eventually depend on their comparison with large instruments. If a small instrument is capable of predicting with sufficient accuracy the optical effects observed with a large instrument, then we are satisfied; however the comparison between different class III instruments may actually give us false results; we may find good agreement, and yet it may mean nothing once we compare them with larger instruments. In case they do not agree, then, we will have to compare them with large instruments anyhow in order to see which of the two is reliable.

WiLson. - A suggestion has been made, and perhaps it can be discussed to-morrow in a more formal way, that a pool of site-survey instruments be set up. Observatories use these survey instruments usually only once, and then put them away or loan them to others who are making surveys. It might be one approach towards standardization of instruments to create a pool of standard survey instruments under the I. A. U., which would be available on loan to whoever wishes to conduct a site survey. These would be primarily class III and II instruments.

There is next the question of personal versus impersonal instruments, particularly in class III observations.

MeINeL. - I would simply like to point out that while a visual test such as the Danjon's test appears to give very reliable results in the hands of well-trained astronomers, in actual practice our recent expe- 
rience in the United States indicates that the visual methods invariably give apparent seeing approximately three or four times better than is indicated by the impersonal instruments at the same time and on the same location. This suggests that there is real problem with visual tests, when it is necessary to rely upon relatively unskilled observers.

Miss UNDERHILL. - I suggest that the explanation of the difference between what you call impersonal measurements and the Danjon test in experienced hands (or inexperienced hands) lies in the process the data goes through. An inexperienced operator looks and takes a guess at the first time he sees the image, while an experienced person knows that he has to look some time at an image before he is really seeing it. So again we are up against the problem of comparing not only our varied data but also the processing system it goes through. If we can ensure that the processing system as well as the incoming data are the same, we can intercompare the data that come out; when we are not sure that we have got the same computing system and the same incoming data, we really have nothing to intercompare.

Courtès. - I think that if you get something quite different with impersonal method and the Danjon method, it is because in your case you are very sensitive to the image deviation of a small telescope (photoelectric telescope). Telescopes of small aperture using Danjon method will always give excellent diffraction rings. The comparison, to be valid, must be made with larger aperture telescopes.

Wilson. - We must pass over completely the question of indirect techniques, which is a large subject involving techniques such as Dr. Lynds', wind tunnels and so on. Perhaps these are questions for a future symposium. Let us pass on to the third part : problems of normalisation of sky brightness and transparency observations.

STock. - The importance of sky brightness for astronomical work has already been stressed a number of times. The background brightness, together with the seeing, gives the practical limiting magnitude and for that reason it is very important to have the sky background as low as possible. In investigating the sky background, we have to divide the problem into two different parts. Firstly, the brightness of the sky background during the bright period of the Moon, when we deal essentially with light scattered in our own atmosphere; except when we are dealing with optimum observatories, we can in all cases neglect scattered artificial light. When we measure the sky background during moonless nights, we are dealing primarily with the background of unresolved stars, galaxies and so on. Superimposed on this, at least 
in certain parts of the sky, we may have other phenomena such as air glow, and this may show a variation from one place to another. So to find the optimum place for work on the faintest stars, we will have to minimise these. The sky background is supposed to be of the order of 21 or 22 magnitudes per square second of arc; however, if you want to check on this, how are you going to do it ? You may select a certain part in a diaphragm of a certain size; but you must be absolutely sure what you have in the diaphragm. For instance, if you have a field of view of I minute of arc, then your sky background corresponds to a I3th magnitude star. A I-minute diaphragm is already rather large. Yet, it shows you that the sky deflection is quite small, so that it requires rather sensitive equipment to measure it with some accuracy. In addition, it shows you that if you happen to have one i 3 th magnitude star in the field, the deflection is already falsified by a factor 2. If you have a $40 \mathrm{~cm}$ telescope, you may still be able to see a 13 th magnitude star, although it will be very faint. You may have ten i 5.5 magnitude stars, none of which you will see, though they will be in the field of view, and they will also falsify the deflection by a factor 2 . In other words, we have to select areas for sky background measurements. Since we need a standard for this, we must pick out areas very carefully, we must specify with great precision which diaphragm has to be used, as well as their exact coordinates, if we want to compare sky brightness in various sites. I think this is about all that can be said about desiderata, as far as sky background measurements are concerned on dark nights.

On bright nights, we are limited by the scattered moonlight, and it would mean, at least in the larger observatories, a great re-ordering of programmes if we could extend our limiting magnitude, at full Moon, by one or two magnitudes. This is to say, the light scattered by the Moon is a subject worthy of investigation. The particles scattering responsible for will also affect extinction measurements; however an increase of extinction of a few hundredths of magnitude will correspond to an enormous change in the brightness of the sky background. Extinction measurements alone are thus too insensitive. We must therefore measure scattered light during the lunar period and this is rather a complicated process because the sky brightness depends on the phase of the Moon and on its position. Scattered moonlight is highly polarized. There are many factors to be taken into account and we can agree on a practical method only if we do a great deal of standardization. I want to present one proposal which has not been realized yet, but which may give you some idea of how this could be done. Since the sky background is rather bright, we do not have to bother too much about the background of stars except for bright stars. What we could do, is to take a small telescope and point to the zenith. You can forget 
about the optics; you may even take the objective out, so that you just have a long tube, pointed to the sky with a photometer at the bottom. Then, you measure the sky brightness at the zenith for certain zenith distances of the Moon, for instance, 60,45 and $30^{\circ}$, or any selection of zenith distances that seem to be practical. You can make a very simple shadowcasting device so that the observer will always know without any calculation when he has to measure the sky background deflection. Then, to analyse this, you make a plot of the magnitude versus the phase of the Moon; you expect to get a curve with a maximum about the full Moon; of course, near new Moon you get zero. You will probably find a large scatter of points, the lowest values corresponding to pure Rayleigh scattering. So one would expect all the points to fall within an envelope which could then be used as a standard. After each measurement you enter with the phase, you look at what you have measured, you determine its deviation from this minimum, and this gives you the increase of the sky background due to scattered moonlight. This is a fairly simplified reduction method and it should give you results very quickly (').

Craldea. - This may depend, I think, on the condensation of the water vapor. In one part of the month, the Moon is visible in the morning, when there is more condensed water, which scatters light more than the air.

STock. - This method does not specify at all what the source of the scattering is. It may be droplets, but we do not really care what it is.

Fournier D'AlBe. - It occurs to me that it would be worthwhile to give a brief summary of the instruments which are used for measuring the intensity of sunlight and the sky brightness during the day. There are three types of instruments.

Firstly those which measure the light coming from a limited solid angle, usually $5^{\circ}$. There is one absolute instrument, the Angström pyrheliometer, and a number of second-order instruments of which probably the most commonly used is the actinometer with a Moll thermopile. I suppose a hundred of these instruments are in use in meteorological services but a great deal of work done during the Geophysical year is available on microcards from the W. M. 0 .

The second type of instrument, called a solarimeter, measures the total radiation received on a horizontal surface from Sun and sky. It can be used either on its own or in conjunction with a second instrument fitted with a metal band which shades the thermopile from direct sunlight. With a pair of solarimeters you can measure in this way,

(1) Note de l'Éditeur. - Une méthode d'observation tout à fait analogue a été proposée dans le Rapport du Groupe de Travail (Voir p. 3i6). 
not only the total radiation, but also that received from the sky, and that from the sun itself. You may thus calculate the ratio between scattered sky light and direct sunlight. This gives you in fact the contrast between the Sun and the sky, and therefore an indication of that between stars and the sky. This instrument costs about 200 and is easily portable. Lastly, a rough measure of the intensity of direct sunlight and therefore of the scattering power of the atmosphere, can be obtained from the ordinary sunshine recorder of the CampbellStokes type normally used only for recording the number of hours of sunshine. Some work in Mexico shows that there is a relation between the loss of weight of the paper-strip used in this instrument and the total incoming direct radiation throughout a day, which can thus be estimated with an accuracy of plus or minus ro $\%$. This is a simple way of getting an indication of atmospheric opacity. There are hundreds of these instruments in use all over the world and probably they would be quite suitable for field surveys.

Hoag. - If the cloud conditions are the same day and night, do you feel any information on the night scattering conditions can be made on the basis of day observations ?

Fournier D'Albe. - In general, yes, since the total amount of scattering material between the observer and the top of the atmosphere remains approximately the same, even though its vertical distribution changes between day and night. We cannot of course separate the secondary scattering of light reflected from clouds. But I think it is probably fairly small.

Stock. - The experience that I have is that at times you can, at times you cannot. For instance, on the Chilean coast during the summer, the inversion layer which is below the top of the mountain breaks down, let us say around midday or early afternoon, and then you get convection carrying a great deal of scattering particles up to a very great altitude above your site and this of course affects very much the scattering properties of the sky. However this scattering material sinks down again below the site during the late afternoon. If you confine yourself to times when the inversion is still there, you probably get measurements which allow you to draw valid conclusions concerning the night. However, once you have convection reaching above your site, you can no longer use daytime measurements.

Fracastoro. - I would like to ask how can you measure seeing with a 6-inch telescope?

RöscH. - Again, what do you call seeing? I suppose in this case it means difference between the real diffraction pattern and the theoretical one. May I say that for a $15 \mathrm{~cm}$ objective which most of the time 
(except in extremely bad conditions when it is absolutely useless to do anything) is smaller than the Gamma regions, as Courtès called them, then the diffraction pattern is not very different from the theoretical one and in this sense there is no " seeing " effect at all.

Wilson. - Topic 4, the frequency and duration of site testing observations, was discussed in part by several speakers earlier in the week. The last topic is problems of data, reduction and comparison. This includes one question raised earlier in the afternoon, a discussion of the reduction of seeing observations to the zenith. Is someone prepared to speak on this particular topic?

Siedentopf. - I would like to draw a representation of data, that would seem to me ideal, but I am not quite sure it can be achieved. On the horizontal line I put the different months of the year. On the vertical, the number of observing hours, where image diameter is smaller than a certain amount. For instance, let us begin with $0.3^{\prime \prime}$, then for instance $0.6^{\prime \prime}$ and so on until the end. That gives us the total number of observing hours for each month of the year. If we have these data for a certain site, we would be able to decide on the programme for the year. We must of course bear in mind that we always use a sampling technique. We are only able to make statistical estimates from our sampling, so we get very diffuse limits between the different ranges of seeing quality. However, such a representation may give an idea of the character of certain observing sites.

WiLson. - One or two other matters have been suggested by the committee, for consideration or discussion by the group. One is that there have been several papers recently, which it would be very valuable to have appear in the proceedings of this Symposium. If it were possible to include an appendix of such papers, it might be useful to publish them. Another request is for a bibliography on this subject to be prepared and included as an appendix to this Symposium.

Rösch. - In reply to the first point raised by Dr. Wilson, I think that it may be difficult to include in the proceedings of this Symposium papers which have not been specially prepared for it. I do not know what would be the reaction of Dr. Sadler. I am sure he will agree to publish all that we have said here, but I am not sure he would accept to have this increased by papers which may appear somewhere else. On the second point, I agree that it would be extremely valuable to compile a bibliography, but who will do the job ? I shall consider how it may be done for inclusion in the publication of this Symposium, provided that it does not delay too much the publication. 
Kiepenheuer. - I guess that if you collect the references at the end of all the introductory papers which have been presented here, they will cover at least $80 \%$ of the total possible bibliography.

Rösch. - This is a good suggestion, although I am not sure they will cover $80 \%$.

Now, I want to come to a more general point. Gentlemen, I think we have said a great deal this week, but there is somebody who has still to speak to-morrow : this is the Chairman. It will not be easy for me to make a summary of all what we have said. I shall do my best to-morrow to mention the points on which it seems to me we have reached agreement, the points which appear to be important and need more work, and finally those which we were supposed to examine but which we have not discussed at all. I have already a list of such points, but I am sure I shall forget some. So, to save the situation, may I ask you, if you have in mind some important problem which has been overlooked in our sessions, to be kind enough to write it on a small paper (not more than two lines) and give me the paper to morrow at 9.3o. I shall try to take into account all the suggestions, or maybe I shall just read out the list of the papers received ! 